mitted drug resistance associated mutations and HIV-1 subtypes in new HIV-1 diagnoses, U.S.-2006. AIDS 2010;24:1203-1212.

6. Eron JJ, Young B, Cooper DA, et al. Switch to a raltegravir-based regimen versus continuation of a lopinavir-ritonavir-based regimen in stable HIV-infected patients with suppressed viraemia (SWITCHMRK 1 and 2): two multicentre, double-blind, randomised controlled trials. Lancet 2010;375:396-407.

7. Barnes S, Loutfy M, Prasad R, Tan DHS. Institutional policies and physician prescribing practices of non-occupational postexposure prophylaxis (nPEP) in Ontario. In: 21st Canadian Conference on HIV/AIDS Research; 2012; Montreal, Canada. Abstract P158.

8. Kunches LM, Meehan TM, Boutwell RC, McGuire JF. Survey of nonoccupational HIV postexposure prophylaxis in hospital emergency departments. I Acquir Immune Defic Syndr 2001;26:263265.

\section{Reply to Tan et al}

To the Editor-In the letter by Tan et al, ${ }^{1}$ in response to the updated US Public Health Service (PHS) guidelines, ${ }^{2}$ several issues are raised for consideration by institutions when developing their protocols for occupational exposures to human immunodeficiency virus (HIV). We emphasize that the US PHS guidelines are not intended to be used as a strict protocol; they are open to interpretation and modification, based on local circumstances. The PHS working group and expert consultant panel used available scientific evidence and expert opinion as the basis for developing the updated guidelines. However, evidence of superior efficacy of a single PEP regimen among the preferred and alternatives ${ }^{2}$ does not exist and is unlikely to be developed. Demonstrating differential efficacy among PEP regimens that likely possess a similar ability to prevent infection is limited by both the low HIV transmission rate associated with occupational exposures as well as the ethical considerations associated with conducting a randomized controlled trial in that setting. Thus, most of the opinion expressed in the guideline was based on relevant but indirect evidence. The expert panel believed that the regimen adherence advantages of a raltegravir (RAL)-based regimen offered a slight benefit over similar regimens containing protease inhibitors. An optimal single PEP regimen for occupational exposures has not been demonstrated and, given the constraints noted above, likely never will be.

We agree that evidence of PEP tolerability and adherence are among the factors that should inform PEP regimen choices. Tan et al $^{1}$ describe clinical outcome data ${ }^{1,3-5}$ among RAL- and lopinivir/ritonavir-based PEP regimen recipients who primarily experienced nonoccupational exposures. Though the authors suggest roughly comparable outcomes, we interpret these data differently. The $15 \%$ higher average regimen completion rate among RAL-based PEP regimen recipients seems to indicate a slight advantage of RAL-based
PEP. We nonetheless recommend caution when extrapolating from data describing primarily nonoccupational PEP recipients to the occupational setting. Historically, healthcare personnel taking occupational PEP have reported much higher rates of regimen intolerance than persons taking these agents for either nonoccupational exposures or as treatment for infection; ${ }^{6}$ thus, one might expect different PEP completion rates between nonoccupational and occupational exposure populations.

Tan et $\mathrm{al}^{1}$ question the benefit of the minimal drug interactions afforded by RAL-based PEP regimens and indicate that significant polypharmacy is uncommon among their PEP recipients. Minimizing the risk for drug interactions can increase medication adherence and acceptance. Taking even a single medication (either prescription or over the counter) while receiving PEP can place a PEP recipient at risk for significant drug interactions. Because RAL can be administered with proton pump inhibitors, $\mathrm{H} 2$ blockers, antidepressants, and oral contraceptives, all of which are commonly used by relatively healthy personnel, we believe that RALbased regimens might have a relative advantage. PEP regimen adherence rarely exceeds $85 \%$ in most published studies, suggesting that adherence remains a significant issue. Thus, addressing factors that can improve adherence is likely to increase effectiveness.

The commentary authors suggest caution with the use of the tenofovir, emtricitabine, and RAL regimen as PEP for exposures to source patients known or suspected to harbor viruses resistant to nucleotide reverse-transcriptase inhibitors. We agree-and the guidelines indicate-that special considerations should be given to circumstances in which exposure to resistant virus is likely. Expert consultation is recommended for exposures to known or suspected drug-resistant HIV to ensure that drugs to which the source virus is unlikely to be resistant are prescribed as PEP. ${ }^{2}$ The relevance of RAL's modest genetic barrier to resistance in the treatment of HIV infection may not be directly applicable to the success of PEP. PEP efficacy data remain too limited to indicate whether or how genetic barriers to resistance influence HIV PEP outcomes.

Tan et $\mathrm{al}^{1}$ question whether simplification of clinical decision making by eliminating exposure risk stratification may be less relevant to occupational PEP and suggest that occupational exposures are often managed in institutional corporate health clinics by expert occupational health providers. Occupational health clinics may provide management for exposures that occur in outpatient and inpatient settings when exposures occur during the daytime hours during which occupational health clinics are typically open. However, such occupational health clinics are unlikely to be available for individuals sustaining exposures outside these normal clinic hours. For facilities that provide 24 -hour patient care-such as acute care hospitals, long-term acute care hospitals, skilled nursing facilities, and emergency treatment centers-occupational exposures to bloodborne pathogens occur at all 
hours of the day. Our experience suggests that after-hours exposures are often managed in emergency rooms or standalone clinics, and physicians in these settings may be less familiar with the approaches to exposure management and pharmacologic agents for prophylaxis.

Finally, we agree with Tan et $\mathrm{al}^{1}$ that medication cost is an important consideration, and the guidelines indicate that a more cost-efficient alternative to RAL may be required. ${ }^{2}$ Individual facilities should consider undertaking comparative cost-benefit analyses-emphasizing factors that improve PEP adherence and minimize toxicities-when updating institutional PEP policies and protocols. The guidelines list several alternative medications for PEP regimens. ${ }^{2}$

Other experts are in agreement with PHS on a preference for RAL-based occupational PEP. ${ }^{7}$ Given the limited data available on PEP administration, efficacy, and failures, some experts may disagree, and reasonable arguments can be made to support different conclusions. We echo the call for publication of relevant PEP data to inform regimen decisions. While such data are unlikely to coalesce around a single optimal regimen, electronic publication of this guideline is intended to allow for prompt updates when additional data become available.

\section{ACKNOWLEDGMENTS}

The findings and conclusions in this report are those of the authors and do not necessarily represent the official position of the Centers for Disease Control and Prevention, the Food and Drug Administration, or the National Institutes of Health.

Potential conflicts of interest. All authors report no conflicts of interest relevant to this article. All authors submitted the ICMJE Form for Disclosure of Potential Conflicts of Interest, and the conflicts that the editors consider relevant to this article are disclosed here.

\section{David T. Kuhar, MD; ${ }^{1}$ Kimberly A. Struble, PharmD; ${ }^{2}$} David K. Henderson, $\mathrm{MD}^{3}$

Affiliations: 1. Division of Healthcare Quality Promotion, National Center for Emerging and Zoonotic Infectious Diseases, Centers for Disease Control and Prevention, Atlanta, Georgia; 2. Division of Antiviral Products, Center for Drug Evaluation and Research, Food and Drug Administration, Silver Spring, Maryland; 3. Office of the Deputy Director for Clinical Care, Clinical Center, National Institutes of Health, Bethesda, Maryland.

Address correspondence to David T. Kuhar, MD, Division of Healthcare Quality Promotion, National Center for Emerging and Zoonotic Infectious Diseases, Centers for Disease Control and Prevention, 1600 Clifton Road, NE, MS A-31, Atlanta, GA 30333 (jto7@cdc.gov).

Infect Control Hosp Epidemiol 2014;35(3):328-329

This article is in the public domain, and no copyright is claimed. $0899-823 \mathrm{X}$ 2014/3503-0023. DOI: $10.1086 / 675350$

\section{REFERENCES}

1. Tan DHS, Goddey-Erikefe B, Yoong D, et al. Selecting an antiretroviral regimen for human immunodeficiency virus postexposure prophylaxis in the occupational setting. Infect Control Hosp Epidemiol 2013;35:326-328.

2. Kuhar DT, Henderson DK, Struble KA, et al. Updated US Public
Health Service guidelines for the management of occupational exposures to human immunodeficiency virus and recommendations for postexposure prophylaxis. Infect Control Hosp Epidemiol 2013;34:875-892.

3. Mayer KH, Mimiaga MJ, Gelman M, Grasso C. Raltegravir, tenofovir DF, and emtricitabine for postexposure prophylaxis to prevent the sexual transmission of HIV: safety, tolerability, and adherence. J Acquir Immune Defic Syndr 2012;59:354-359.

4. McAllister J, Read P, McNulty A, Tong W, Ingersoll A, Carr A. Raltegravir-emtricitabine-tenofovir as HIV nonoccupational post-exposure prophylaxis in men who have sex with men: safety, tolerability and adherence. HIV Med 2014;15:13-22.

5. Tosini W, Muller P, Prazuck T, et al. Tolerability of HIV postexposure prophylaxis with tenofovir/emtricitabine and lopinivir/ ritonavir tablet formation. AIDS 2010;24:2375-2380

6. Henderson DK. Human immunodeficiency virus in healthcare settings. In: Mandell GL, Bennett JE, Dolin R, eds. Principles and Practice of Infectious Diseases. 7th ed. New York: Churchill Livingstone, 2009;3753-3770.

7. New York State Department of Health AIDS Institute. HIV Prophylaxis Following Occupational Exposure. New York: New York State Department of Health AIDS Institute, 2012. http://www .hivguidelines.org/clinical-guidelines/post-exposure-prophylaxis /hiv-prophylaxis-following-occupational-exposure/. Accessed November 26, 2013.

\section{Factors Associated with Hand Hygiene Compliance among Critical Care Nurses}

To the Editor-We read with great interest the article by Kowitt et $\mathrm{al}^{1}$ that investigated the factors associated with hand hygiene compliance at a teaching hospital. In this study, they showed that the significant differences in compliance were noted between different professions. The compliance of hand hygiene was higher among nursing staff $(84 \%)$ than among physician staff (78\%) and support staff (69\%). However, we wonder whether there would be other factors-such as age, sex, education, length of employment, and experience in health care-affecting hand hygiene compliance even within the same profession. Therefore, we conducted a study to investigate the possible factors associated with hand hygiene compliance among critical care nurses.

This study was carried out in 5 intensive care units (ICUs) at regional teaching hospitals. There were 63 adult ICU beds, and 150 critical care nurses were employed in the ICU. Compliance was defined as the frequency of the number of performed actions to the number of hand hygiene opportunities. Observation of hand hygiene compliance was carried out by trained members of the nursing department. Between October 1 and October 14,2013, the compliance with hand hygiene (World Health Organization's 5 Moments for Hand Hygiene) among critical care nurses was observed. Ethics approval was obtained from the institution review board of Chi Mei Medical Center. Comparisons between each variable/cat- 